\title{
A STRONGER BERTRAND'S POSTULATE WITH AN APPLICATION TO PARTITIONS
}

\author{
ROBERT E. DRESSLER ${ }^{1}$
}

\begin{abstract}
In this paper we give a stronger form of Bertrand's postulate and use it to prove that every positive integer, except 1,2 , 4,6 , and 9 , can be written as the sum of distinct odd primes.
\end{abstract}

Introduction. The purpose of this paper is to derive, in an elementary manner, a stronger form of Bertrand's postulate and to use this stronger form to prove the following:

THEOREM. Every positive integer, except $1,2,4,6$, and 9 can be written as the sum of distinct odd primes.

1. The stronger form. Bertrand's postulate states that if $p_{n}$ is the $n$th prime then $p_{n+1}<2 p_{n}$ for all $n$. Hardy and Wright [2, p. 343] give a proof of this result due to Erdös and they mention that a modification of the proof will show that $p_{n+1}<2 p_{n}-2$ for all $n>2$. In fact, we note that one can adapt the proof to show that for any positive integer $k$, there exists a positive integer $M$ such that $p_{n+1}<2 p_{n}-k$ if $n>M$. The result we need is this:

LEMMA. $\quad p_{n+1}<2 p_{n}-10$ for all $n>6$.

Proof. We will sketch the proof by indicating the necessary modifications in the proof which appears in [2] and we will use the same notation as [2].

To begin with, assume there is some integer $n \geqq 1000$ such that there is no prime $q$ satisfying $n<q<2 n-10$. For the binomial coefficient $N=\left(\begin{array}{c}2 n-10 \\ n-10\end{array}\right)$ and any prime $p$,

$$
k_{p}=\sum_{m=1}^{\infty}\left(\left[\frac{2 n-10}{p^{m}}\right]-\left[\frac{n}{p^{m}}\right]-\left[\frac{n-10}{p^{m}}\right]\right)
$$

where $k_{p}$ is defined to be the largest power of $p$ which divides $N$. For

Received by the editors May 24, 1971.

AMS 1970 subject classifications. Primary 10J15.

Key words and phrases. Bertrand's postulate, primes, partition.

I I am grateful to Professor L. M. Chawla for telling me of a related result [1] due to him and Yeung which led me to consider this problem.

(c) American Mathematical Society 1972 
$(2 / 3) n<p \leqq n$ we have $k_{p}=0$ except for at most 3 primes $p$ with $n-9 \leqq$ $p \leqq n-5$ where $k_{p}$ is 1 . So

Also,

$$
\sum_{p \mid N} \log p \leqq \sum_{p \leqq(2 / 3) n} \log p+3 \log n<(4 / 3) n \log 2+3 \log n
$$

Thus

$$
\sum_{k_{p} \geqq 2} k_{p} \log p \leqq(2 n-10)^{1 / 2} \log (2 n-10) .
$$

$$
\log N \leqq \frac{4}{3} n \log 2+3 \log n+(2 n-10)^{1 / 2} \log (2 n-10)
$$

But

$$
\begin{aligned}
2^{(2 n-10)} & \leqq(2 n-10)\left(\begin{array}{c}
2 n-10 \\
n-5
\end{array}\right) \\
& =(2 n-10) N\left(\frac{n(n-1)(n-2)(n-3)(n-4)}{(n-5)(n-6)(n-7)(n-8)(n-9)}\right)
\end{aligned}
$$

Taking logarithms of both sides and then applying ( $\left.{ }^{*}\right)$ gives us a contradiction. We then check the prime tables for $n<1000$ to obtain our result.

2. Proof of the theorem. We first notice that from direct calculation we have that if $n \leqq 23$ and $n \neq 1,2,4,6,9$ then $n$ can be written as the sum of distinct odd primes. Suppose for some $n>23$, the conclusion holds for all $m<n, m \neq 1,2,4,6,9$. Let $p$ be the largest prime $\leqq n$. Then write $n=p+$ $(n-p)$.

Case I. If $n-p \neq 1,2,4,6,9$ then we are done by the lemma and the induction hypothesis, since by Bertrand's postulate it follows that $p>$ $n-p$ and so $p$ cannot be a summand in any partition of $n-p$.

Case II. If $n-p=1$ then write $n=p_{1}+\left(p-p_{1}+1\right)$ where $p_{1}$ is the largest prime $<p$. Then we have $p-p_{1}+1<p_{1}-9$ by the lemma. Also $p-p_{1}+1$ is odd and hence $\neq 2,4,6$, and we are done if $p-p_{1}+1 \neq 9$. If $p-p_{1}+1=9$ then write $n=p_{2}+\left(p_{1}-p_{2}+9\right)$ where $p_{2}$ is the largest prime $<p_{1}$. We have $p_{1}-p_{2}+9<p_{2}-1$ by the lemma and since $p_{1}-p_{2}+9>9$ we are done by the induction hypothesis.

Case III. If $n-p=2$ then write $n=p_{1}+\left(p-p_{1}+2\right)$. Now $4 \leqq p-p_{1}+$ $2<p_{1}-8$ and $p-p_{1}+2$ is even so we are done unless $p-p_{1}+2=4$ or 6 . If $p-p_{1}+2=4$ write $n=p_{2}+\left(p_{1}-p_{2}+4\right)$ and we have $8 \leqq p_{1}-p_{2}+4<p_{2}-6$ (because $p_{2}<p_{1}-2$ ). But $p_{1}-p_{2}+4$ is even (and hence $\neq 9$ ) and we are done. If $p_{1}-p_{2}+2=6$ then write $n=p_{2}+\left(p_{1}-p_{2}+6\right)$ and we have $10 \leqq$ $p_{1}-p_{2}+6<p_{2}-4$ so we are done. 
Case IV. If $n-p=4$ then write $n=p_{1}+\left(p-p_{1}+4\right)$. We then have $6 \leqq p-p_{1}+4<p_{1}-6$. Since $p-p_{1}+4$ is even we are done unless $p-p_{1}+4=$ 6 . If this is the case, write $n=p_{2}+\left(p_{1}-p_{2}+6\right)$. Now $8 \leqq p_{1}-p_{2}+6<p_{2}-4$. Also $p_{1}-p_{2}+6$ is even (and hence $\neq 9$ ) and we are done.

Case V. If $n-p=6$ then write $n=p_{1}+\left(p-p_{1}+6\right)$. We have $8 \leqq p-$ $p_{1}+6<p_{1}-4$ and since $p-p_{1}+6$ is even (and hence $\neq 9$ ) we are done.

Case VI. If $n-p=9$ then write $n=p_{1}+\left(p-p_{1}+9\right)$. We have $9<p-$ $p_{1}+9<p_{1}-1$ and we are done.

\section{BIBLIOGRAPHY}

1. L. M. Chawla and C. D. N. Yeung, On an additive arithmetic function and its related partition function, J. Natur. Sci. and Math. (to appear).

2. G. H. Hardy and E. M. Wright, An introduction to the theory of numbers, 4th ed., Oxford Univ. Press, London, 1960.

Department of Mathematics, Kansas State University, Manhattan, Kansas 66502 